\title{
STRONG CONVERGENCE OF AN ITERATIVE ALGORITHM FOR SYSTEMS OF VARIATIONAL INEQUALITIES AND FIXED POINT PROBLEMS IN $q$-UNIFORMLY SMOOTH BANACH SPACES
}

\author{
JAE UG JEONG
}

\begin{abstract}
In this paper, we introduce a new iterative scheme to investigate the problem of finding a common element of nonexpansive mappings and the set of solutions of generalized variational inequalities for a $k$-strict pseudo-contraction by relaxed extra-gradient methods. Strong convergence theorems are established in $q$-uniformly smooth Banach spaces.
\end{abstract}

\section{Introduction}

Throughout this paper, we assume that $E$ is a real Banach space and $E^{*}$ the dual space of $E$. Let $C$ be a subset of $E$ and $T$ be a self mapping of $C$. Denote by Fix $(T)$ the set of fixed points of $T$, that is, $\operatorname{Fix}(T)=\{x \in C: T x=x\}$. When $\left\{x_{n}\right\}$ is a sequence in $E$, $x_{n} \rightarrow x\left(x_{n} \rightarrow x\right)$ will denote strong(weak) convergence of the sequence $\left\{x_{n}\right\}$ to $x$.

Let $q>1$ be a real number. The duality mapping $J_{q}: E \rightarrow 2^{E^{*}}$ is defined by

$$
J_{q}(x)=\left\{f \in E^{*}:\langle x, f\rangle=\|x\|^{q}, \quad\|f\|=\|x\|^{q-1}\right\}, \quad \forall x \in E .
$$

In particular, $J=J_{2}$ is called the normalized duality mapping and $J_{q}(x)=\|x\|^{q-2} J_{2}(x)$ for $x \neq 0$. If $E$ is a Hilbert space, then $J=I$, where $I$ is the identity mapping.

Received April 27, 2012. Revised June 11, 2012. Accepted June 15, 2012.

2010 Mathematics Subject Classification: 41A65, 47J20, 47H09.

Key words and phrases: Strong convergence, $k$-strict pseudo-contraction, $q$ uniformly smooth Banach space, variational inequality. 
Recall that a mapping $T$ is said to be nonexpansive if

$$
\|T x-T y\| \leq\|x-y\|
$$

for all $x, y \in C$. A mapping $T$ is called a pseudo-contraction if there exists some $j_{q}(x-y) \in J_{q}(x-y)$ such that

$$
\left\langle T x-T y, j_{q}(x-y)\right\rangle \leq\|x-y\|^{q}
$$

for all $x, y \in C$. $T$ is said to be a $k$-strict pseudo-contraction in the terminology of Browder and Petryshyn [1] if there exists a constant $k>0$ such that

$$
\left\langle T x-T y, j_{q}(x-y)\right\rangle \leq\|x-y\|^{q}-k\|(I-T) x-(I-T) y\|^{q}
$$

for every $x, y \in C$ and for some $j_{q}(x-y) \in J_{q}(x-y)$.

REMARK 1.1. From (1.1) we can prove that if $T$ is $k$-strict pseudocontraction, then $T$ is Lipschitz continuous with the Lipschitz constant $L=\frac{1+k}{k}$.A Banach space $E$ is called uniformly convex if for each $\varepsilon>0$ there is a $\delta>0$ such that for $x, y \in E$ with $\|x\|,\|y\| \leq 1$ and $\|x-y\| \geq \varepsilon$, $\|x+y\| \leq 2(1-\delta)$ holds. It is known that a uniformly convex Banach space is reflexive and strictly convex. Let $S(E)=\{x \in E:\|x\|=1\}$. $E$ is said to be Gâteaux differentiable if the limit

$$
\lim _{t \rightarrow 0} \frac{\|x+t y\|-\|x\|}{t}
$$

exists for each $x, y \in S(E)$. In this case, $E$ is called smooth. Let $\rho_{E}:[0, \infty) \rightarrow[0, \infty)$ be the modulus of smoothness of $E$ defined by

$$
\rho_{E}(t)=\sup \left\{\frac{1}{2}(\|x+y\|+\|x-y\|)-1: x \in S(E), \quad\|y\| \geq t\right\} .
$$

A Banach space $E$ is said to be uniformly smooth if $\lim _{t \rightarrow 0} \frac{\rho(t)}{t}=0$. Let $q>1$. A Banach space $E$ is said to be $q$-uniformly smooth if there exists a fixed constant $c>0$ such that $\rho(t) \leq c t^{q}$. Recall that construction of fixed points for nonexpansive mappings and $\lambda$-strict pseudocontractions via the Mann's iterative algorithm has been extensively investigated by many authors (see $[3,6,7,8]$ ). The Mann iteration is extensively and successfully used to approximate fixed points of nonexpansive mappings.

However, iterative methods for strict pseudo-constractions are far less developed than for nonexpansive mappings. On the other hand, strict pseudo-contractions have more powerful applications than nonexpansive mappings do in solving inverse problems (see [11]). Therefore it is 
interesting to develop the theory of iterative methods for strict pseudocontractions.In 1967, Halpen [4] introduced the following explicit iteration scheme for a nonexpansive mapping $T$ which was referred to Halpern iteration: for $u, x_{0} \in K, \alpha_{n} \in[0,1]$,

$$
x_{n+1}=\alpha_{n} u+\left(1-\alpha_{n}\right) T x_{n} .
$$

Recently, Zhou [17] obtained strong convergence theorem for the following iterative sequence in a 2-uniformly smooth Banach space $E$ : for $u, x_{0} \in E$ and a $\lambda$-strict pseudo-contraction $T$,

$$
x_{n+1}=\beta_{n} u+\gamma_{n} x_{n}+\left(1-\beta_{n}-\gamma_{n}\right)\left[\alpha_{n} T x_{n}+\left(1-\alpha_{n}\right) x_{n}\right],
$$

where $\left\{\alpha_{n}\right\},\left\{\beta_{n}\right\}$ and $\left\{\gamma_{n}\right\}$ in $(0,1)$ satisfy

(i) $a \leq \alpha_{n} \leq \frac{\lambda}{K^{2}}$ for some $a>0$ and for all $n \geq 0$;

(ii) $\lim _{n \rightarrow \infty} \beta_{n}=0$ and $\sum_{n=1}^{\infty} \beta_{n}=\infty$;

(iii) $\lim _{n \rightarrow \infty}\left|\alpha_{n+1}-\alpha_{n}\right|=0$;

(iv) $0<\liminf _{n \rightarrow \infty} \gamma_{n} \leq \lim \sup _{n \rightarrow \infty} \gamma_{n}<1$.

Very recently, Zhang and Shu [16] extended Zhou's results to $q$-uniformly smooth Banach space.

Motivated and inspired by the above works, in this paper, we consider the problem of convergence of an iterative algorithm for a system of generalized variational inequalities involving strictly pseudo-contractions and a nonexpansive mapping. We prove the strong convergence of purposed iterative scheme in uniformly convex and $q$-uniformly smooth Banach spaces.

\section{Preliminaries}

Let $C$ be a nonempty closed convex subset of a Banach space $E$ and $E^{*}$ the dual space of $E$.

Definition 2.1. Let $E$ be a real Banach space, $C$ a nonempty closed and convex subset of $E$ and $K$ a nonempty subset of $C$. Let $Q$ be a mapping of $C$ into $K$. Then $Q$ is said to be:

(1) sunny if for each $x \in C$ and $t \in[0,1]$ we have

$$
Q(t x+(1-t) x)=Q x
$$

(2) a retraction of $C$ onto $K$ if

$$
Q x=x, \quad \forall x \in K
$$


(3) a sunny nonexpansive retraction if $Q$ is sunny nonexpansive and a retraction onto $K$.

The following result describes a characterization of sunny nonexpansive retractions on a smooth Banach space.

Proposition 2.1. ([9]) Let $E$ be a smooth Banach space and let $K$ be a nonempty subset of $E$. Let $Q: E \rightarrow K$ be a retraction and let $J$ be the normalized duality mapping on $E$. Then the following are equivalent:

(a) $Q$ is sunny and nonexpansive;

(b) $\|Q x-Q y\|^{2} \leq\langle x-y, J(Q x-Q y)\rangle, \quad \forall x, y \in E$;

(c) $\langle x-Q x, J(y-Q x)\rangle \leq 0, \quad \forall x \in E, y \in K$.

Proposition 2.2. ([5]) Let $K$ be a nonempty closed convex subset of a uniformly convex and uniformly smooth Banach space $E$ and $S$ a nonexpansive mapping of $C$ into itself with $\operatorname{Fix}(S) \neq \phi$. Then the set Fix $(S)$ is a sunny nonexpansive retract of $C$. Reich [10], in 1980, proved the following behavior for nonexpansive mappings.

Proposition 2.3. Let $E$ be a real uniformly smooth Banach space and $C$ a nonempty closed convex subset of $E$. Let $T: C \rightarrow C$ be a nonexpansive mapping with a fixed point and let $z \in C$. For each $t \in(0,1)$, let $z_{t}$ be the unique solution of the equation $x=t z+(1-t) T x$. Then $\left\{z_{t}\right\}$ converges to a fixed point of $T$ as $t \rightarrow 0$ and

$$
Q z=s-\lim _{t \rightarrow 0} z_{t}
$$

defines the unique sunny nonexpansive retraction from $C$ onto Fix $(T)$, that is, $Q$ satisfies the property:

$$
\langle u-Q u, J(y-Q u)\rangle \leq 0, \quad \forall u \in C, y \in F i x(T) .
$$

Motivated by Wang and Chen [13], we consider the following general system of variational inequalities in a uniformly smooth Banach space $E$. Let $S: C \rightarrow C$ be a $k$-strict pseudo-contraction. Find $\left(x^{*}, y^{*}\right) \in C \times C$ such that

$$
\begin{cases}\left\langle\lambda(I-S) y^{*}+x^{*}-y^{*}, J\left(x-x^{*}\right)\right\rangle \geq 0, & \forall x \in C, \\ \left\langle\mu(I-S) x^{*}+y^{*}-x^{*}, J\left(x-x^{*}\right)\right\rangle \geq 0, & \forall x \in C .\end{cases}
$$

In order to prove our main results, we need the following lemmas. 
Lemma 2.1. ([14]) Let $E$ be a real q-uniformly smooth Banach space. Then there exists a constant $c_{q}>0$ such that

$$
\|x+y\|^{q} \leq\|x\|^{q}+q\left\langle y, j_{q}(x)\right\rangle+c_{q}\|y\|^{q}
$$

for all $x, y \in E$.

Lemma 2.2. ([12]) Let $\left\{z_{n}\right\}$ and $\left\{w_{n}\right\}$ be two bounded sequences in a Banach space $E$ such that

$$
z_{n+1}=\left(1-\gamma_{n}\right) z_{n}+\gamma_{n} w_{n}, \quad n \geq 1,
$$

where $\left\{\gamma_{n}\right\}$ satisfies condition: $0<\liminf _{n \rightarrow \infty} \gamma_{n} \leq \limsup _{n \rightarrow \infty} \gamma_{n}<1$. If $\lim \sup _{n \rightarrow \infty}\left(\left\|w_{n+1}-w_{n}\right\|-\left\|z_{n+1}-z_{n}\right\|\right) \leq 0$, then $w_{n}-z_{n} \rightarrow 0$ as $n \rightarrow \infty$.

Lemma 2.3. ([2]) Let $C$ be a nonempty closed convex subset of a real Banach space $E$. Let $T_{1}$ and $T_{2}$ be nonexpansive mappings from $C$ into itself with a common fixed point. Define a mapping $T: C \rightarrow C$ by

$$
T x=\delta T_{1} x+(1-\delta) T_{2} x, \quad \forall x \in C,
$$

where $\delta$ is a constant in $(0,1)$. Then $T$ is nonexpansive and Fix $(T)=$ $\operatorname{Fix}\left(T_{1}\right) \cap \operatorname{Fix}\left(T_{2}\right)$.

Lemma 2.4. ([15]) Assume that $\left\{\alpha_{n}\right\}$ is a sequence of nonnegative real numbers such that

$$
\alpha_{n+1} \leq\left(1-\gamma_{n}\right) \alpha_{n}+\delta_{n}
$$

where $\left\{\gamma_{n}\right\}$ is a sequence in $(0,1)$ and $\left\{\delta_{n}\right\}$ is a sequence such that

(a) $\sum_{n=1}^{\infty} \gamma_{n}=\infty$;

(b) $\limsup _{n \rightarrow \infty} \frac{\delta_{n}}{\gamma_{n}} \leq 0$ or $\sum_{n=1}^{\infty}\left|\delta_{n}\right|<\infty$. Then $\lim _{n \rightarrow \infty} \alpha_{n}=0$.

Lemma 2.5. For given $\left(x^{*}, y^{*}\right) \in C \times C$, where $y^{*}=Q_{C}\left(x^{*}-\mu(I-\right.$ $\left.S) x^{*}\right),\left(x^{*}, y^{*}\right)$ is a solution of problem (2.1) if and only if $x^{*}$ is a fixed point of the mapping $D: C \rightarrow C$ defined by

$$
D(x)=Q_{C}\left[Q_{C}(x-\mu(I-S) x)-\lambda(I-S) Q_{C}(x-\mu(I-S) x)\right], \quad \forall x \in C,
$$

where $\lambda, \mu>0$ are constants and $Q_{C}$ is a sunny nonexpansive retraction from $E$ onto $C$.

Proof. Observe that

$$
\begin{cases}\left\langle\lambda(I-S) y^{*}+x^{*}-y^{*}, J\left(x-x^{*}\right)\right\rangle \geq 0, & \forall x \in C, \\ \left\langle\mu(I-S) x^{*}+y^{*}-x^{*}, J\left(x-x^{*}\right)\right\rangle \geq 0, & \forall x \in C .\end{cases}
$$


$\Leftrightarrow$

$$
\left\{\begin{array}{l}
x^{*}=Q_{C}\left(y^{*}-\lambda(I-S) y^{*}\right), \\
y^{*}=Q_{C}\left(x^{*}-\mu(I-S) x^{*}\right) .
\end{array}\right.
$$

$\Leftrightarrow$

$$
x^{*}=Q_{C}\left[Q_{C}\left(x^{*}-\mu(I-S) x^{*}\right)-\lambda(I-S) Q_{C}\left(x^{*}-\mu(I-S) x^{*}\right)\right] .
$$

\section{Main results}

Now, we consider the following main result of this paper.

TheOrem 3.1. Let $C$ be a nonempty closed convex subset of a uniformly convex and q-uniformly smooth Banach space $E$ and $Q_{C}$ a sunny nonexpansive retraction from $E$ onto $C$. Let $S: C \rightarrow C$ be a $k$-strict pseudo-contraction such that $F i x(S) \neq \phi$ and $T: C \rightarrow C$ a nonexpansive mapping with Fix $(T) \neq \phi$. Assume that $F=F i x(T) \cap F i x(D) \neq \phi$, where $D$ is defined as Lemma 2.5. Let a sequence $\left\{x_{n}\right\}$ be generated by

$$
\left\{\begin{array}{l}
x_{1}=u \in C \\
y_{n}=Q_{C}\left(x_{n}-\mu(I-S) x_{n}\right) \\
x_{n+1}=\alpha_{n} u+\beta_{n} x_{n}+\gamma_{n}\left[\delta T x_{n}+(1-\delta) Q_{C}\left(y_{n}-\lambda(I-S) y_{n}\right)\right], n \geq 1
\end{array}\right.
$$

where $\delta \in(0,1), \lambda, \mu \in\left(0, \min \left\{1,\left(\frac{q k}{c_{q}}\right)^{\frac{1}{q-1}}\right\}\right]$ and $\left\{\alpha_{n}\right\},\left\{\beta_{n}\right\}$ and $\left\{\gamma_{n}\right\}$ are sequences in $[0,1]$ such that

(H1) $\alpha_{n}+\beta_{n}+\gamma_{n}=1, \quad \forall n \geq 1$,

(H2) $\lim _{n \rightarrow \infty} \alpha_{n}=0, \sum_{n=1}^{\infty} \alpha_{n}=\infty$;

(H3) $0<\liminf _{n \rightarrow \infty} \beta_{n} \leq \limsup _{n \rightarrow \infty} \beta_{n}<1$.

Then $\left\{x_{n}\right\}$ defined by (3.1) converges strongly to $\bar{x}=Q_{F} u$ and $(\bar{x}, \bar{y})$, where $\bar{y}=Q_{C}(\bar{x}-\mu(I-S) \bar{x})$ and $Q_{F}$ is the unique sunny nonexpansive retraction from $C$ onto $F$, is a solution of the problem (2.1).

Proof. We divide our proofs into several steps as follows.

(Step 1.) First, we show that $F$ is closed and convex.

It is well known that Fix $(T)$ is closed and convex. Next, we show that Fix $(D)$ is closed and convex. For any $\lambda, \mu \in(0, M], M=\min \left\{1,\left(\frac{q k}{c_{q}}\right)^{\frac{1}{q-1}}\right\}$, 
we have that the mappings $I-\mu(I-S)$ and $I-\lambda(I-S)$ are nonexpansive mappings. Indeed, from Lemma 2.1, we have for all $x, y \in C$,

$$
\begin{aligned}
\| & (I-\lambda(I-S)) x-(I-\lambda(I-S)) y \|^{q} \\
= & \|x-y-\lambda(x-y-(S x-S y))\|^{q} \\
\leq & \|x-y\|^{q}-q \lambda\left\langle x-y-(S x-S y), J_{q}(x-y)\right\rangle \\
& +c_{q} \lambda^{q}\|x-y-(S x-S y)\|^{q} \\
\leq & \|x-y\|^{q}-q \lambda\|x-y\|^{q}+q \lambda\left\langle S x-S y, J_{q}(x-y)\right\rangle \\
& +c_{q} \lambda^{q}\|x-y-(S x-S y)\|^{q} \\
\leq & \|x-y\|^{q}-q \lambda\|x-y\|^{q}+q \lambda\left[\|x-y\|^{q}-k\|(I-S) x-(I-S) y\|^{q}\right] \\
& +c_{q} \lambda^{q}\|x-y-(S x-S y)\|^{q} \\
= & \|x-y\|^{q}-\lambda\left(q k-c_{q} \lambda^{q-1}\right)\|x-y-(S x-S y)\|^{q} \\
\leq & \|x-y\|^{q},
\end{aligned}
$$

which shows that $I-\lambda(I-S)$ is a nonexpansive mapping. So is $I-$ $\mu(I-S)$. By Lemma 2.5, we can see that

$$
\begin{aligned}
D & =Q_{C}\left[Q_{C}(I-\mu(I-S))-\lambda(I-S) Q_{C}(I-\mu(I-S))\right] \\
& =Q_{C}(I-\lambda(I-S)) Q_{C}(I-\mu(I-S))
\end{aligned}
$$

is nonexpansive. Thus, $F=F i x(T) \cap F i x(D)$ is closed and convex.

(Step 2.) The sequences $\left\{x_{n}\right\}$ is bounded.

For $x^{*} \in F=F i x(T) \cap F i x(D)$, we have that

$$
x^{*}=Q_{C}\left[Q_{C}\left(x^{*}-\mu(I-S) x^{*}\right)-\lambda(I-S) Q_{C}\left(x^{*}-\mu(I-S) x^{*}\right)\right] .
$$

Set $y^{*}=Q_{C}\left(x^{*}-\mu(I-S) x^{*}\right)$. We obtain $x^{*}=Q_{C}\left(y^{*}-\lambda(I-S) y^{*}\right)$. Since $y_{n}=Q_{C}\left(x_{n}-\mu(I-S) x_{n}\right)$, we have

$$
\begin{aligned}
\left\|y_{n}-y^{*}\right\| & =\| Q_{C}\left(x_{n}-\mu(I-S) x_{n}\right)-Q_{C}\left(x^{*}-\lambda(I-S) x^{*} \|\right. \\
& \leq\left\|x_{n}-x^{*}\right\| .
\end{aligned}
$$

For the sake of simplicity, let $u_{n}=\delta T x_{n}+(1-\delta) Q_{C}\left(y_{n}-\lambda(I-S) y_{n}\right)$ for each $n \geq 1$. By (3.2), we have

$$
\begin{aligned}
\left\|u_{n}-x^{*}\right\|= & \left\|\delta T x_{n}+(1-\delta) Q_{C}\left(y_{n}-\lambda(I-S) y_{n}\right)-x^{*}\right\| \\
\leq & \delta\left\|T x_{n}-x^{*}\right\| \\
& +(1-\delta) \| Q_{C}\left(y_{n}-\lambda(I-S) y_{n}-Q_{C}\left(y^{*}-\lambda(I-S) y^{*}\right) \|\right. \\
\leq & \delta\left\|x_{n}-x^{*}\right\|+(1-\delta)\left\|y_{n}-y^{*}\right\| \\
\leq & \delta\left\|x_{n}-x^{*}\right\|+(1-\delta)\left\|x_{n}-x^{*}\right\| \\
= & \left\|x_{n}-x^{*}\right\| .
\end{aligned}
$$


Then we have

$$
\begin{aligned}
\left\|x_{n+1}-x^{*}\right\| & =\left\|\alpha_{n} u+\beta_{n} x_{n}+\gamma_{n} u_{n}-x^{*}\right\| \\
& \leq \alpha_{n}\left\|u-x^{*}\right\|+\beta_{n}\left\|x_{n}-x^{*}\right\|+\gamma_{n}\left\|u_{n}-x^{*}\right\| \\
& \leq \alpha_{n}\left\|u-x^{*}\right\|+\left(1-\alpha_{n}\right)\left\|x_{n}-x^{*}\right\| \\
& \leq \max \left\{\left\|u-x^{*}\right\|,\left\|x_{n}-x^{*}\right\|\right\} .
\end{aligned}
$$

By induction, we get

$$
\left\|x_{n}-x^{*}\right\| \leq \max \left\{\left\|u-x^{*}\right\|,\left\|x_{1}-x^{*}\right\|\right\} .
$$

Thus, $\left\{x_{n}\right\}$ is bounded, so are $\left\{y_{n}\right\}$ and $\left\{u_{n}\right\}$.

(Step 3.) $x_{n+1}-x_{n} \rightarrow 0$ as $n \rightarrow \infty$.We now define $w_{n}=\frac{x_{n+1}-\beta_{n} x_{n}}{1-\beta_{n}}$.

Set $M_{1}=\|u\|+\sup \left\{\left\|u_{n}\right\|\right\}$. By using (3.1), we get

$$
\begin{aligned}
\left\|w_{n+1}-w_{n}\right\|= & \left\|\frac{\alpha_{n+1} u+\gamma_{n+1} u_{n+1}}{1-\beta_{n+1}}-\frac{\alpha_{n} u+\gamma_{n} u_{n}}{1-\beta_{n}}\right\| \\
= & \|\left(\frac{\alpha_{n+1}}{1-\beta_{n+1}}-\frac{\alpha_{n}}{1-\beta_{n}}\right) u+\left(\frac{\alpha_{n+1}}{1-\beta_{n+1}}-\frac{\alpha_{n}}{1-\beta_{n}}\right) u_{n} \\
& -\left(\frac{\alpha_{n+1}}{1-\beta_{n+1}}-\frac{\alpha_{n}}{1-\beta_{n}}\right) u_{n}+\frac{\gamma_{n+1}}{1-\beta_{n+1}} u_{n+1}-\frac{\gamma_{n}}{1-\beta_{n}} u_{n} \| \\
\leq & \left|\frac{\alpha_{n+1}}{1-\beta_{n+1}}-\frac{\alpha_{n}}{1-\beta_{n}}\right|\left(\|u\|+\left\|u_{n}\right\|\right)+\frac{\gamma_{n+1}}{1-\beta_{n+1}}\left\|u_{n+1}-u_{n}\right\| \\
\leq & M_{1}\left|\frac{\alpha_{n+1}}{1-\beta_{n+1}}-\frac{\alpha_{n}}{1-\beta_{n}}\right|+\left\|u_{n+1}-u_{n}\right\|
\end{aligned}
$$

and

$$
\begin{aligned}
\left\|u_{n+1}-u_{n}\right\|= & \| \delta T x_{n+1}+(1-\delta) Q_{C}\left(y_{n+1}-\lambda(I-S) y_{n+1}\right) \\
& -\left(\delta T x_{n}+(1-\delta) Q_{C}\left(y_{n}-\lambda(I-S) y_{n}\right)\right) \| \\
\leq & \delta\left\|T x_{n+1}-T x_{n}\right\| \\
& +(1-\delta)\left\|Q_{C}\left(y_{n+1}-\lambda(I-S) y_{n+1}\right)-Q_{C}\left(y_{n}-\lambda(I-S) y_{n}\right)\right\| \\
\leq & \delta\left\|x_{n+1}-x_{n}\right\|+(1-\delta)\left\|y_{n+1}-y_{n}\right\| \\
\leq & \delta\left\|x_{n+1}-x_{n}\right\|+(1-\delta)\left\|x_{n+1}-x_{n}\right\| \\
= & \left\|x_{n+1}-x_{n}\right\| .
\end{aligned}
$$

Substituting (3.5) into (3.4) yields

$$
\left\|w_{n+1}-w_{n}\right\| \leq M_{1}\left|\frac{\alpha_{n+1}}{1-\beta_{n+1}}-\frac{\alpha_{n}}{1-\beta_{n}}\right|+\left\|x_{n+1}-x_{n}\right\| .
$$

By the assumptions on $\left\{\alpha_{n}\right\}$ and $\left\{\beta_{n}\right\}$, we get

$$
\limsup _{n \rightarrow \infty}\left(\left\|w_{n+1}-w_{n}\right\|-\left\|x_{n+1}-x_{n}\right\|\right) \leq 0 .
$$


By using Lemma 2.2, we conclude that $w_{n}-x_{n} \rightarrow 0$ as $n \rightarrow \infty$. Noting that $x_{n+1}-x_{n}=\left(1-\beta_{n}\right)\left(w_{n}-x_{n}\right)$, we get $x_{n+1}-x_{n} \rightarrow 0$ as $n \rightarrow \infty$.

(Step 4.) There exists a continuous path $\left\{x_{t}\right\}$ such that $x_{t} \rightarrow \bar{x}$ as $t \rightarrow 0$, where $\bar{x}=Q_{F} u$ and $Q_{F}: C \rightarrow F$ is the unique sunny nonexpansive retraction from $C$ onto $F$. Define a mapping $T_{\delta}: C \rightarrow C$ by

$$
T_{\delta} x=\delta T x+(1-\delta) Q_{C}(I-\lambda(I-S)) Q_{C}(I-\mu(I-S)) x, \quad \forall x \in C .
$$

Then $T_{\delta}$ is nonexpansive and

$$
\begin{aligned}
F i x\left(T_{\delta}\right) & =F i x(T) \cap F i x\left(Q_{c}(I-\lambda(I-S)) Q_{C}(I-\mu(I-S))\right) \\
& =F i x(T) \cap F i x(D) \\
& =F
\end{aligned}
$$

by Lemma 2.3. For $t \in(0,1)$ we define a contraction via

$$
T_{\delta}^{t} x=t u+(1-t) T_{\delta} x, \quad \forall x \in C .
$$

Then, the Banach contraction mapping principle ensures that there exists a unique path $x_{t} \in C$ such that

$$
x_{t}=t u+(1-t) T_{\delta} x_{t}
$$

for every $t \in(0,1)$. By Proposition 2.3, we know that $x_{t} \rightarrow \bar{x} \in \operatorname{Fix}\left(T_{\delta}\right)$ as $t \rightarrow \infty$. Further, if we define $Q_{\text {Fix }\left(T_{\delta}\right)} u=\bar{x}$, then $Q_{F i x\left(T_{\delta}\right)}: C \rightarrow F i x\left(T_{\delta}\right)$ is a unique sunny nonexpansive retraction from $C$ onto $F i x\left(T_{\delta}\right)$. Noting that $\operatorname{Fix}\left(T_{\delta}\right)=F$, we see that $Q_{F}: C \rightarrow F$ is indeed the unique sunny nonexpansive retraction from $C$ onto $F$.

(Step 5.) $\lim \sup _{n \rightarrow \infty}\left\langle u-\bar{x}, J\left(x_{n}-\bar{x}\right)\right\rangle \leq 0$, where $\bar{x}=Q_{F} u$.

We note that

$$
\begin{aligned}
\left\|x_{n}-T_{\delta} x_{n}\right\| & \leq\left\|x_{n}-x_{n+1}\right\|+\left\|x_{n+1}-T_{\delta} x_{n}\right\| \\
& \leq\left\|x_{n}-x_{n+1}\right\|+\alpha_{n}\left\|u-T_{\delta} x_{n}\right\|+\beta_{n}\left\|x_{n}-T_{\delta} x_{n}\right\| .
\end{aligned}
$$

This implies that

$$
\left(1-\beta_{n}\right)\left\|x_{n}-T_{\delta} x_{n}\right\| \leq\left\|x_{n}-x_{n+1}\right\|+\alpha_{n}\left\|u-T_{\delta} x_{n}\right\| .
$$

It follows from conditions (H2), (H3) and Step 3 that $x_{n}-T_{\delta} x_{n} \rightarrow 0$ as $n \rightarrow \infty$. Since

$$
\begin{aligned}
x_{t}-x_{n} & =t u+(1-t) T_{\delta} x_{t}-x_{n} \\
& =(1-t)\left(T_{\delta} x_{t}-x_{n}\right)+t\left(u-x_{n}\right),
\end{aligned}
$$


then

$$
\begin{aligned}
\left\|x_{t}-x_{n}\right\|^{2}= & (1-t)\left\langle T_{\delta} x_{t}-x_{n}, J\left(x_{t}-x_{n}\right)\right\rangle+t\left\langle u-x_{n}, J\left(x_{t}-x_{n}\right)\right\rangle \\
= & (1-t)\left[\left\langle T_{\delta} x_{t}-T_{\delta} x_{n}, J\left(x_{t}-x_{n}\right)\right\rangle+\left\langle T_{\delta} x_{n}-x_{n}, J\left(x_{t}-x_{n}\right)\right\rangle\right] \\
& +t\left\langle u-x_{t}, J\left(x_{t}-x_{n}\right)\right\rangle+t\left\langle x_{t}-x_{n}, J\left(x_{t}-x_{n}\right)\right\rangle \\
\leq & (1-t)\left(\left\|x_{t}-x_{n}\right\|^{2}+\left\|T_{\delta} x_{n}-x_{n}\right\|\left\|x_{t}-x_{n}\right\|\right) \\
& +t\left\langle u-x_{t}, J\left(x_{t}-x_{n}\right)\right\rangle+t\left\|x_{t}-x_{n}\right\|^{2} \\
= & \left\|x_{t}-x_{n}\right\|^{2}+\left\|T_{\delta} x_{n}-x_{n}\right\|\left\|x_{t}-x_{n}\right\|+t\left\langle u-x_{t}, J\left(x_{t}-x_{n}\right)\right\rangle .
\end{aligned}
$$

It turns out that

$$
\left\langle x_{t}-u, J\left(x_{t}-x_{n}\right)\right\rangle \leq \frac{1}{t}\left\|T_{\delta} x_{n}-x_{n}\right\|\left\|x_{t}-x_{n}\right\|, \quad \forall t \in(0,1) .
$$

By the above inequality, we have

$$
\limsup _{n \rightarrow \infty}\left\langle x_{t}-u, J\left(x_{t}-x_{n}\right) \leq 0 .\right.
$$

Since $J$ is strong to weak* uniformly continuous on bounded subset of $E$, we see that

$$
\begin{aligned}
\mid & \left\langle u-\bar{x}, J\left(x_{n}-\bar{x}\right)\right\rangle-\left\langle x_{t}-u, J\left(x_{t}-x_{n}\right)\right\rangle \mid \\
\leq & \left|\left\langle u-\bar{x}, J\left(x_{n}-\bar{x}\right)\right\rangle-\left\langle u-\bar{x}, J\left(x_{n}-x_{t}\right)\right\rangle\right| \\
& +\left|\left\langle u-\bar{x}, J\left(x_{n}-x_{t}\right)\right\rangle-\left\langle x_{t}-u, J\left(x_{t}-x_{n}\right)\right\rangle\right| \\
= & \left|\left\langle u-\bar{x}, J\left(x_{n}-\bar{x}\right)-J\left(x_{n}-x_{t}\right)\right\rangle\right|+\left|\left\langle x_{t}-\bar{x}, J\left(x_{n}-x_{t}\right)\right\rangle\right| \\
\leq & \|u-\bar{x}\|\left\|J\left(x_{n}-\bar{x}\right)-J\left(x_{n}-x_{t}\right)\right\|+\left\|x_{t}-\bar{x}\right\|\left\|x_{n}-x_{t}\right\| \\
\rightarrow & 0 \quad \text { as } \quad t \rightarrow 0 .
\end{aligned}
$$

For any $\varepsilon>0$, there exists $\delta>0$ such that for every $t \in(0, \delta)$

$$
\left\langle u-\bar{x}, J\left(x_{n}-\bar{x}\right)\right\rangle \leq\left\langle x_{t}-u, J\left(x_{t}-x_{n}\right)\right\rangle+\varepsilon .
$$

Therefore

$$
\limsup _{n \rightarrow \infty}\left\langle u-\bar{x}, J\left(x_{n}-\bar{x}\right)\right\rangle \leq \limsup _{n \rightarrow \infty}\left\langle x_{t}-u, J\left(x_{t}-x_{n}\right)\right\rangle+\varepsilon .
$$

This implies that

$$
\limsup _{n \rightarrow \infty}\left\langle u-\bar{x}, J\left(x_{n}-\bar{x}\right)\right\rangle \leq 0
$$


(Step 6.) $x_{n} \rightarrow \bar{x} \in Q_{F} u$ as $n \rightarrow \infty$. By using (3.3) we have

$$
\begin{aligned}
\left\|x_{n+1}-\bar{x}\right\|^{2}= & \left\langle\alpha_{n} u+\beta_{n} x_{n}+\gamma_{n} u_{n}-\bar{x}, J\left(x_{n+1}-\bar{x}\right)\right\rangle \\
= & \alpha_{n}\left\langle u-\bar{x}, J\left(x_{n+1}-\bar{x}\right)\right\rangle+\beta_{n}\left\langle x_{n}-\bar{x}, J\left(x_{n+1}-\bar{x}\right)\right\rangle \\
& +\gamma_{n}\left\langle u_{n}-\bar{x}, J\left(x_{n+1}-\bar{x}\right)\right\rangle \\
\leq & \alpha_{n}\left\langle u-\bar{x}, J\left(x_{n+1}-\bar{x}\right)\right\rangle+\beta_{n}\left\|x_{n}-\bar{x}\right\|\left\|x_{n+1}-\bar{x}\right\| \\
& +\gamma_{n}\left\|u_{n}-\bar{x}\right\| x_{n+1}-\bar{x} \| \\
\leq & \alpha_{n}\left\langle u-\bar{x}, J\left(x_{n+1}-\bar{x}\right)\right\rangle+\beta_{n}\left\|x_{n}-\bar{x}\right\|\left\|x_{n+1}-\bar{x}\right\| \\
& +\gamma_{n}\left\|x_{n}-\bar{x}\right\|\left\|x_{n+1}-\bar{x}\right\| \\
\leq & \alpha_{n}\left\langle u-\bar{x}, J\left(x_{n+1}-\bar{x}\right)\right\rangle+\left(1-\alpha_{n}\right)\left\|x_{n}-\bar{x}\right\|\left\|x_{n+1}-\bar{x}\right\| \\
\leq & \alpha_{n}\left\langle u-\bar{x}, J\left(x_{n+1}-\bar{x}\right)\right\rangle+\frac{1-\alpha_{n}}{2}\left(\left\|x_{n}-\bar{x}\right\|^{2}+\left\|x_{n+1}-\bar{x}\right\|^{2}\right),
\end{aligned}
$$

which implies that

$$
\left\|x_{n+1}-\bar{x}\right\|^{2} \leq\left(1-\alpha_{n}\right)\left\|x_{n}-\bar{x}\right\|^{2}+2 \alpha_{n}\left\langle u-\bar{x}, J\left(x_{n+1}-\bar{x}\right)\right\rangle
$$

and hence $x_{n} \rightarrow \bar{x}$ as $n \rightarrow \infty$ by virtue of Lemma 2.4. This completes the proof.

REMARK 3.1. Since $L^{p}(1<p \leq 2)$ is uniformly convex and $p$ uniformly smooth, we see that Theorem 3.1 is applicable to $L^{p}$ for $1<p \leq 2$.

\section{Applications}

In real Hilbert spaces, Lemma 2.3 is reduced to the following.

LEMMA 4.1. Let $C$ be a nonempty closed convex subset of a Hilbert space $H$. For given $(\bar{x}, \bar{y}) \in C \times C$, where $\bar{y}=P_{C}(\bar{x}-\mu(I-S) \bar{x}),(\bar{x}, \bar{y})$ is a solution of the following problem:

$$
\begin{cases}\langle\lambda(I-S) \bar{y}+\bar{x}-\bar{y}, x-\bar{x}\rangle \geq 0, & \forall x \in C, \\ \langle\mu(I-S) \bar{x}+\bar{y}-\bar{x}, x-\bar{x}\rangle \geq 0, & \forall x \in C,\end{cases}
$$

if and only if $\bar{x}$ is a fixed point of the mapping $\bar{D}: C \rightarrow C$ defined by

$$
\bar{D}(x)=P_{C}\left[P_{C}(x-\mu(I-S) x)-\lambda(I-S) P_{C}(x-\mu(I-S) x)\right],
$$

where $P_{C}$ is a metric projection $H$ onto $C$. Utilizing Theorem 3.1 we can obtain the following results. 
Theorem 4.1. Let $C$ be a nonempty closed convex subset of a Hilbert space $H$. Let $S: C \rightarrow C$ be a $k$-strict pseudo-contraction such that $\operatorname{Fix}(S) \neq \phi$ and $T: C \rightarrow C$ a nonexpansive mapping with Fix $(T) \neq \phi$. Assume that $F=F i x(T) \cap F i x(\bar{D}) \neq \phi$, where $\bar{D}$ is defined as Lemma 4.1. Let a sequence $\left\{x_{n}\right\}$ be generated by

$$
\left\{\begin{array}{l}
x_{1}=u \in C \\
y_{n}=P_{C}\left(x_{n}-\mu(I-S) x_{n}\right) \\
x_{n+1}=\alpha_{n} u+\beta_{n} x_{n}+\gamma_{n}\left[\delta T x_{n}+(1-\delta) P_{C}\left(y_{n}-\lambda(I-S) y_{n}\right)\right], \quad n \geq 1
\end{array}\right.
$$

where $\delta \in(0,1), \lambda, \mu \in(0,2 k)$ and $\left\{\alpha_{n}\right\},\left\{\beta_{n}\right\}$ and $\left\{\gamma_{n}\right\}$ are sequences in $[0,1]$ such that

(H1) $\alpha_{n}+\beta_{n}+\gamma_{n}=1, \quad \forall n \geq 1$,

(H2) $\lim _{n \rightarrow \infty} \alpha_{n}=0, \sum_{n=1}^{\infty} \alpha_{n}=\infty$,

(H3) $0<\liminf _{n \rightarrow \infty} \beta_{n} \leq \lim \sup _{n \rightarrow \infty} \beta_{n}<1$.

Then $\left\{x_{n}\right\}$ defined by (4.2) converges strongly to $\bar{x}=P_{F} u$ and $(\bar{x}, \bar{y})$ is a solution of problem (4.1), where $\bar{y}=P_{C}(\bar{x}-\mu(I-S) \bar{x})$.

Theorem 4.2. Let $C$ be a nonempty closed convex subset of $H$. Let $T, S: C \rightarrow C$ be a nonexpansive mapping such that Fix $(T) \neq \phi$ and Fix $(S) \neq \phi$. Assume that $F=F i x(T) \cap F i x(\bar{D}) \neq \phi$, where $\bar{D}$ is defined as Lemma 4.1. Let the sequence $\left\{x_{n}\right\}$ generated by (4.2) such that the conditions (H1), (H2), (H3) hold. Then $\left\{x_{n}\right\}$ converges strongly to $\bar{x}=P_{F} u$ and $(\bar{x}, \bar{y})$ is a solution of problem (4.1), where $\bar{y}=P_{C}(\bar{x}-\mu(I-S) \bar{x})$.

\section{References}

[1] F.E. Browder and W.V. Petryshyn, Construction of fixed points of nonlinear mappings in Hilbert spaces, J. Math. Anal. Appl. 20 (1967), 197-228.

[2] R.E. Bruck, Properties of fixed point sets of nonexpansive mappings in Banach spaces, Trans. Amer. Math. Soc. 179 (1973), 251-262.

[3] C.E. Chidume and S. A. Mutangadura, An example on the Mann iteration method for Lipschitz pseudocontractions, Proc. Amer. Math. Soc. 129 (2001), 2359-2363.

[4] B. Halpern, Fixed points of nonexpansive maps, Bull. Amer. Math. Soc. 73(1967), 957-961.

[5] S. Kitahara and W. Takahashi, Image recovery by convex combinations of sunny nonexpansive retractions, Topol. Methods Nonlinear Anal. 2 (1993), 333-342.

[6] W.R. Mann, Mean value methods in iterations, Proc. Amer. Math. Soc. 14(1953), 506-510. 
[7] G. Marino and H. K. Xu, Weak and strong convergence theorems for strict pseudo-contractions in Hilbert spaces, J. Math. Anal. Appl. 329 (2007), 336-346.

[8] K. Nakajo and W. Takahashi, Strong convergence theorems for nonexpansive mappings and nonexpansive semigroups, J. Math. Anal. Appl. 279 (2003), 372-379.

[9] S. Reich, Asymptotic behavior of constractions in Banach spaces, J. Math. Anal. Appl. 44(1973), 57-70.

[10] S. Reich, Strong convergence theorems for resolvents of accretive operators in Banach spaces, J. Math. Anal. Appl. 75 (1980), 287-292.

[11] O. Scherzer, Convergence criteria of iterative methods based on Landweber iteration for solving nonlinear problems, J. Math. Anal. Appl. 194 (1991), 911-933.

[12] T. Suzuki, Strong convergence of Krasnoselskii and Manns type sequences for one parameter nonexpansive semigroups without Bochner integrals, J. Math. Anal. Appl. 305 (2005), 227-239.

[13] Y. Wang and R. Chen, Hybrid methods for accretive variational inequalities involving pseudocontractions in Banach spaces, Fixed Point Theory Appl. 63 (2011). doi:10.1186/1687-1812-2011-63.

[14] H.K. Xu, Inequalities in Banach spaces with applications, Nonlinear Anal. 16 (1991), 1127-1138.

[15] H.K. Xu, Iterative algorithms for nonlinear operators, J. Lond. Math. Soc. 66 (2002), 240-256.

[16] H. Zhang and Y.Su, Convergence theorems for strict pseudo-contractions in q-uniformly smooth Banach spaces, Nonlinear Anal. 71 (2009), 4572-4580.

[17] H. Zhou, Convergence theorems for $\lambda$-strict pseudocontractions in 2uniformly smooth Banach spaces, Nonlinear Anal. 69 (2008), 3160-3173.

Department of Mathematics

Dongeui University

Busan 614-714, South Korea

E-mail: jujeong@deu.ac.kr 